\title{
Detachment of Extensor Carpi Ulnaris and Anterior Branches of the Radial Collateral Ligament from the Epicondyle: A Modification of Kocher Lateral Approach for the Treatment of Radial Head Fractures
}

\author{
Georgios Touloupakis ${ }^{1}$ Elena Biancardi ${ }^{1}$ Stefano Ghirardelli ${ }^{10}$ Guido Antonini ${ }^{10}$ \\ Cornelio Crippa ${ }^{10}$ \\ ${ }^{1}$ Department of Orthopedics and Traumatology, San Carlo Borromeo \\ Hospital, Milan, Italy \\ Address for correspondence Georgios Touloupakis, MD, Via Privata \\ Joints 2019;7:31-36. \\ Don Bartolomeo Grazioli 24, 20161 Milano, Italy \\ (e-mail: yorgostoulou@gmail.com).
}

\begin{abstract}
Purpose The aim of this study is to present clinical results of a retrospective case series that includes patients treated with an extensive modification of the Kocher lateral approach to the elbow with surgical detachment of the anterior branches of the radial collateral ligament.

Methods From January 2016 to January 2018, nine patients with closed isolated displaced or multifragmentary radial head fractures (Mason type II, III, or IV) who underwent osteosynthesis or arthroplasty through a modified Kocher lateral elbow approach were available for follow-up.

Keywords

- radial head fractures

- lateral approach to the elbow

- lateral collateral ligament

- radial collateral ligament

Results There were six female and three male patients. The median age at the time of surgery was 52 years old (range: $26-70$ ). The dominant upper limb was injured in $22.2 \%$ of patients. After a median follow-up of 8 (range: $6-27$ ) months, all patients regained completely all their daily activities and no cases of infections were recorded.

Conclusion We believe that the approach proposed can be a useful choice to deal with these challenging injuries. Our strategy may represent a valid alternative to more popular approaches as the use of anchors decreases the risk of instability that is the major danger considered in the past when soft tissues as tendons and ligaments are detached.

Level of Evidence This is a level IV study (therapeutic case series).
\end{abstract}

\section{Introduction}

Treatment of displaced and multifragmentary fractures of the radial head is often quite challenging for the surgeons. Stable fixation is mandatory and the level of difficulty in the surgical management increases if anteromedial involvement of the radial head is present. When the radial head cannot be reconstructed, its replacement is recommended. Careful evaluation of collateral ligaments of the elbow should be considered so as to choose the most accurate treatment and to optimize the surgical outcome.

received

March 21, 2018

accepted after revision

August 7, 2019

published online

October 11, 2019
DOI https://doi.org/

10.1055/s-0039-1697613. ISSN 2282-4324.
There are different approaches to treat radial head fractures but the choice depends on surgeon experience and specific fracture characteristics. The approaches involved more frequently in the treatment of these fractures are the Kocher approach and the Kaplan approach. The extensor splitting approach has gained popularity as it provides a better access to the anterior half of the radial head.

The aim of this study it to present clinical results of a retrospective case series that includes patients treated with an extensive modification of the Kocher lateral approach to the elbow with surgical detachment of the anterior branches

Copyright (c) 2019 Georg Thieme Verlag License terms KG Stuttgart · New York

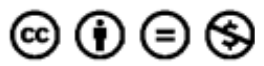


of the radial collateral ligament $(\mathrm{RCL})$. We, therefore, highlight the possibility of an extensive view of the radial head through this surgical window.

\section{Methods}

This retrospective case series reviewed patients with closed isolated displaced or multifragmentary radial head fractures (Mason type II, III, or IV) who underwent osteosynthesis or arthroplasty through a modified Kocher lateral elbow approach. Radial head replacement was performed in the cases with comminuted articular fractures, for which reduction and stable osteosynthesis was extremely demanding.

A total of nine patients had been operated in the orthopaedic surgical unit of the San Carlo Borromeo Hospital (Milan, Italy) from January 2016 to January 2018. Minimum follow-up was 6 months. The X-ray images of all the eligible patients were obtained from the Picture Archiving and Communication System of our institute and examined in at least two standard projections (anteroposterior and lateral radiographic views of the elbow). In addition, a computed tomography scan of the elbow was performed preoperatively in all of the cases. The radial head fractures were divided according to the Mason classification system. The median age of the patients and the side involved were reported.

\section{Surgical Technique}

All procedures were performed under general anesthesia. Patients were positioned supine with the upper limb on a radiolucent table. The elbow was flexed and fully pronated on a hand table to increase the distance between the site of the surgical incision and the posterior interosseous nerve (PIN). Surgical procedures were performed using a modification of the Kocher lateral approach.

A longitudinal skin incision was made directly over the radial head, extending from the lateral epicondyle along the anconeus muscle. The common extensor fascia was visualized and incised. The space between the extensor carpi ulnaris (ECU) and anconeus muscles was identified and the two muscles were separated by a fatty thin connective tissue (-Fig. 1A). Dissection between these muscles was performed. The origin of the ECU was detached from the lateral epicondyle (-Fig. 1B). Anterior retraction was favored. The anconeus muscle was retracted posteriorly without release of its proximal insertion. The lateral collateral ligament complex (LCLC) was identified. The LCLC consists of the annular ligament and the RCL. The lateral ulnar collateral ligament (LUCL) represents the posterior branch of the RCL. The anterior branches of the RCL were detached from the epicondyle. Proximal insertion of RCL was detached; the fibers were reinforced with suture construct and they were divaricated distally. The LUCL was preserved. Muscle retractors were not placed with excessive force to avoid injury of the PIN.

A final control view using the image intensifier was obtained to check the procedure.

A suture anchor (DePuy Synthes Mitek Rotator Cuff QUICKANCHOR size 2 green Ethibond braided polyester suture, 36' [Mitek SportsMedicine, Massachusetts, United States]) double armed with $\mathrm{CP}-2$ needles was applied to the lateral epicondyle and to the ECU with the anterior branches of the RCL being reattached.

At the end of the procedure, the capsule was repaired and the skin was closed using Nylon 2-0 Donati-Allgower suture or by using metal staples.

A brachial-metacarpal cast with the forearm in pronation was applied in every patient at the end of the surgery for 7 days. The patients were allowed to begin gentle passive motion exercises of the shoulder and the elbow the day after the removal of the cast. As a prophylactic therapy of heterotopic ossifications, all patients received $25 \mathrm{mg}$ of indomethacin 3 times a day and $40 \mathrm{mg}$ of omeprazole once a day in the first 3 postoperative weeks.

Physiotherapy was continued after the discharge of the patient on the outpatient department for all the necessary period.
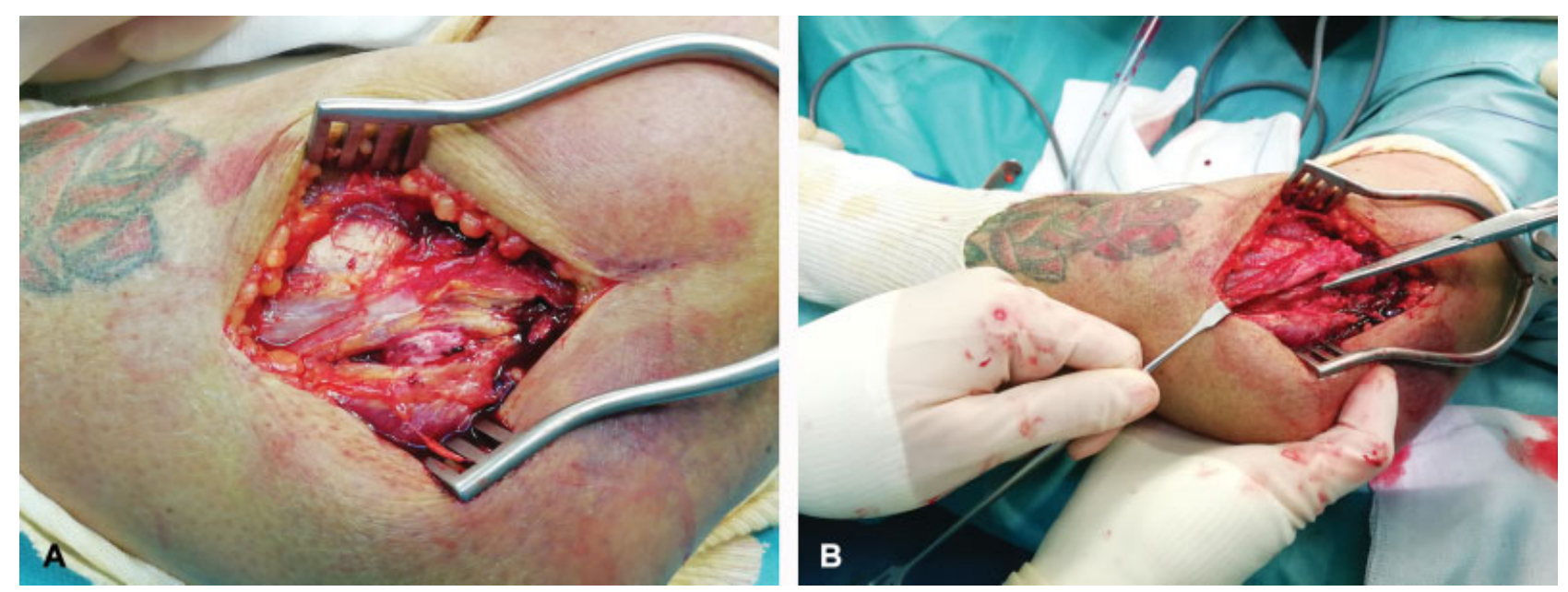

Fig. 1 A 72-year-old female presenting a Mason IV radial head fracture. (A) The space between the extensor carpi ulnaris and anconeus muscles was identified and the two muscles were separated by a fatty thin connective tissue. (B) The origin of the extensor carpi ulnaris was detached from the lateral epicondyle and the anterior branches of the radial collateral ligament were identified. 


\section{Outcome Measurements}

All subjects were followed up as outpatients with X-ray control scans according to our protocol (anteroposterior and lateral projection of the humerus) at 4 weeks, 3 months, 6 months, and thereafter at regular intervals depending on the overall clinical status and necessity of further control. After each follow-up, a proof clinical examination was obtained. Clinical outcome and complications, such as infections and nerve lesions, were examined in the immediate postoperative period. The functional outcome was quantified using the Mayo Elbow Performance (MEP) index.

\section{Results}

The demographic data are illustrated in - Table 1. There were six female and three male patients. The median age at the time of surgery was 52 years old (range: $26-70$ ). The dominant upper limb was injured in $22.2 \%$ of patients. None of the patients had any neurological or vascular deficit at the time of the first clinical examination. One patient had a distal radial articular fracture in the ipsilateral side. All were closed fractures.

After a median follow-up of 8 (range: 6-27) months, all patients regained completely all their daily activities and no cases of infections were recorded.

One patient showed a peripheral nerve palsy of the PIN after surgery due to intraoperative maneuvers; during the follow-up, a gradual improvement was seen and a complete recover was evaluated 6 weeks after surgery.

The median delay from admission to operation was 6 days (range: 3-12 days).

The three radial head replacements were well positioned and no overstuffing was observed. Satisfactory reduction and fixation were achieved in the six open reduction and internal fixation patients (-Fig. 2). Two cases of heterotopic ossifications (Class 1 Hastings and Graham classification: ossification without functional limitation) were recorded, but clinical assessment of the patients was good. No patients required a revision surgery. The mean values of the MEP index scores determined at the last follow-up are reported in -Table 1.

\section{Discussion}

Several surgical approaches to the radial head have been described in the literature. ${ }^{1}$

The original Kocher approach provides extensive access to the elbow. It is typically extended in the interval between the ECU and the anconeus muscles and consists of a subperiosteal release of the LCL extensors from the lateral epicondyle and release of the anconeus muscle, which is retracted posteriously. $^{2}$ In the limited Kocher approach, the interval between ECU and anconeus muscles is prepared without release of adjacent anatomical structures. In the modified Kocher approach, the ECU was detached and a capsular incision was made along the anterior border of the LCL without detaching the proximal fibers of the ligament. The Kaplan approach is a splitting approach between the extensor digitorum communis (EDC) and the extensor carpi radialis brevis and longus muscles, but the PIN is in close proximity, so care must be taken to protect the nerve during this surgical procedure. ${ }^{3}$ In the EDC splitting approach, the EDC tendon is identified and the fibers are split. The capsule and the annular ligament are incised anteriorly to visualize the LUCL that must not be injured in this approach. In the extended EDC splitting approach, the anterior half of the EDC and the extensor carpi radialis are detached from the supracondylar ridge. ${ }^{4,5}$

The surgical approach proposed in our case series is a safe and valid choice for the treatment of complex radial head fractures providing with a good functional outcome. All patients reported satisfactory restoration of range of motion and we did not record in our series complications like instability (posterolateral rotatory instability or varus posteromedial instability) or infection at the last follow-up. This procedure is user-friendly as the lateral approach to the elbow can be easily done to visualize fragments of the radial head.

Table 1 Patients data set (baseline characteristics and functional outcome)

\begin{tabular}{|l|l|l|l|l|l|l|l|l|l|}
\hline Patient & Age & Gender & Dominance & Mason & $\begin{array}{l}\text { Time to } \\
\text { surgery (d) }\end{array}$ & Surgery & $\begin{array}{l}\text { Operative } \\
\text { time }\end{array}$ & $\begin{array}{l}\text { Follow-up } \\
\text { (mo) }\end{array}$ & \begin{tabular}{l} 
MEPS \\
\hline 1
\end{tabular} \\
\hline 70 & F & No & II & 12 & Fixation & 45 & 27 & Excellent \\
\hline 2 & 26 & M & Yes & III & 12 & Fixation & 75 & 24 & Good \\
\hline 3 & 52 & F & No & III & 8 & Replacement & 60 & 18 & Excellent \\
\hline 4 & 39 & M & Yes & IV & 7 & Fixation & 120 & 13 & Excellent \\
\hline 5 & 32 & F & No & III & 4 & Fixation & 145 & 8 & Excellent \\
\hline 6 & 46 & M & No & III & 3 & Fixation & 145 & 6 & Good \\
\hline 7 & 59 & F & No & IV & 6 & Fixation & 55 & 6 & Good \\
\hline 8 & 65 & F & No & IV & 6 & Replacement & 60 & 6 & Excellent \\
\hline 9 & 62 & F & No & 5 & Replacement & 75 & 6 & Good \\
\hline
\end{tabular}

Abbreviation: MEPS, Modified Elbow Performance Score. 

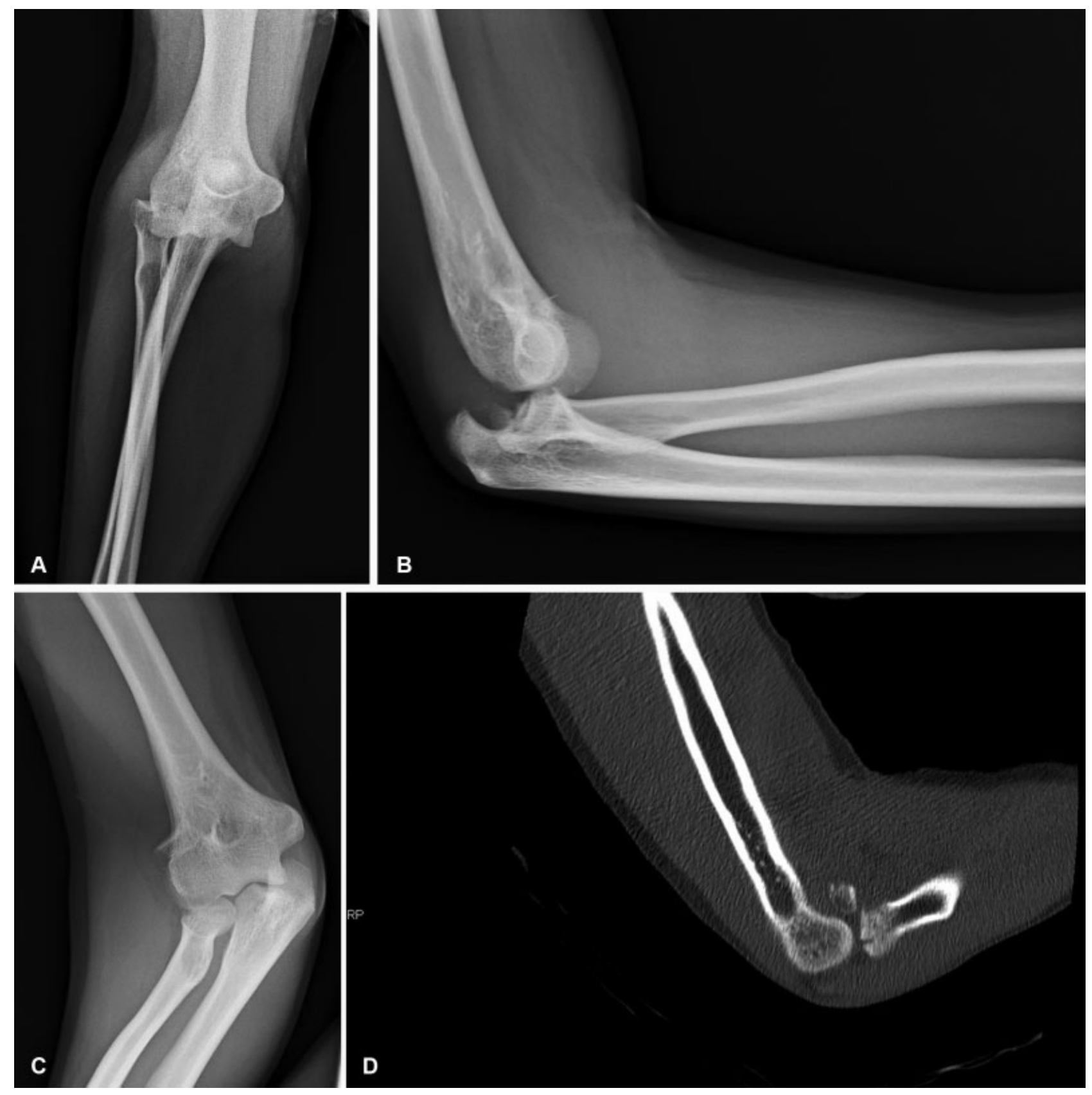

Fig. 2 Initial and postoperative X-rays of a 39-year-old male presenting a Mason IV radial head fracture after high energy trauma. Radiographs at the 12th month follow-up showing a good fracture healing. A-B-C: Post-injury X-rays; D: CT slice of the right elbow; E-F: post-operative X-rays; G-H Twelve months follow-up X-rays.

In our experience, the visualization of the anteromedial surface of the radial head was increased when compared with the limited Kocher approach as proximal detachment of the RCL facilitates maneuver of lateral dislocation of the proximal radius.

The RCL provides varus stability to the humeroradial joint and promotes stability by maintaining the distance between humeral and radial surfaces. In the literature, it is reported that releasing the RCL with and intact LUCL does not promote the above-mentioned instability pattern. ${ }^{6}$

Complementary components of the lateral ligament complex as the accessory lateral collateral ligament and the annular ligament increase stability of the radial head.
Other ligament structures that contribute to stability in the lateral side of the elbow are the quadrate ligament and the oblique cord, anatomical structures poorly visualized in traumatic elbows.

The LUCL that was not violated in our approach is considered to be the main constraint to varus and rotator laxity. Detachment of the ECU increases visualization of the articular surface and simultaneously decreases the necessity of stressing with the retractors the soft tissues around the extensor group of the elbow and so it avoids traction of the PIN.

Refixation of the ECU and RCL with an anchor results in sufficient restoration of varus stability. ECU is a primary 

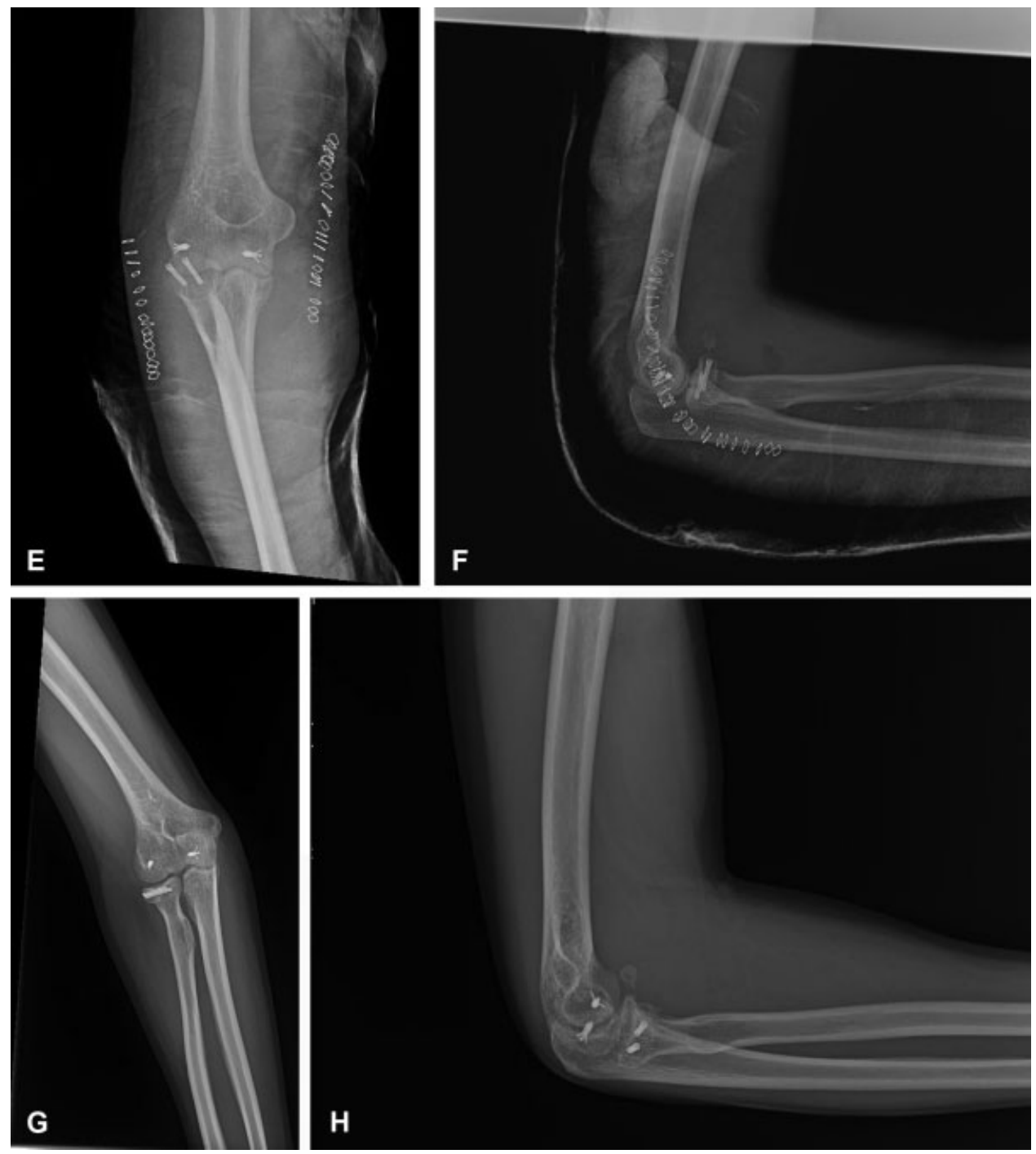

Fig. 2 (Continued)

stabilizer of the elbow joint against the varus forces and stability is strengthened if we consider the fact that anconeus muscle is not disturbed during our approach. Refixation is facilitated from the fact that both the structures are close to each other and formation of scar tissue in the postoperative period increases stability.

The posterolateral rotator stability is provided by intact LUCL, which was not intersected and was not detached during our approach in this case series.

In cases with posterolateral rotator stability as elbow extends, patient becomes apprehensive or radial head dislocates. Despite the fact that the anterior branches of the RCL are tightened when the elbow is extended and the LUCL is loosened during elbow extension and vice versa, it seems that reconstruction with anchors of the RCL and of the ECU is sufficient to prevent posterolateral instability that occurs during last degrees of extension of the elbow. ${ }^{7}$

A limit of our study was the short-term follow-up period. Although the follow-up can be considered short-medium term, we want to remember the results of a study by Giannicola et al. ${ }^{8}$ He has shown how the recovery of ROM after osteosynthesis of complex elbow fractures is acquired in the first 6 postoperative months and that subsequent sessions of physical therapy are not beneficial.

The primary limitation of our study was the limited and heterogeneous number of cases. Furthermore, only the MEP index was used as outcome measure for functional evaluation.

In conclusion, we believe that the approach proposed can be a useful choice to deal with these challenging injuries. Our strategy may represent a valid alternative to more popular approaches as the use of anchors decreases the risk of instability that is the major danger considered in the past when soft tissues as tendons and ligaments are detached.

Conflict of Interest

None declared.

\section{References}

1 Cheung EV, Steinmann SP. Surgical approaches to the elbow. J Am Acad Orthop Surg 2009;17(05):325-333 
36 Modification of Kocher Lateral Approach for the Treatment of Radial Head Fractures Touloupakis et al.

2 Bartoníček J, Naňka O, Tuček M. [Kocher approach to the elbow and its options]. Rozhl Chir 2015;94(10):405-414

3 Han F, Lim CT, Lim JC, Tan BH, Shen L, Kumar VP. Deep branch of the radial nerve in lateral surgical approaches to the radial head - a cadaveric study. Orthop Traumatol Surg Res 2016;102(04):453-458

4 Han F, Teo AQ, Lim JC, Ruben M, Tan BH, Kumar VP. Outcomes using the extensor digitorum communis splitting approach for the treatment of radial head fractures. J Shoulder Elbow Surg 2016;25(02):276-282

5 Desloges W, Louati H, Papp SR, Pollock JW. Objective analysis of lateral elbow exposure with the extensor digitorum communis split compared with the Kocher interval. JBone Joint Surg Am 2014;96(05):387-393

6 Stroyan M, Wilk KE. The functional anatomy of the elbow complex. JOrthop Sports Phys Ther 1993;17(06):279-288

7 Hackl M, Bercher M, Wegmann K, Müller LP, Dargel J. Functional anatomy of the lateral collateral ligament of the elbow. Arch Orthop Trauma Surg 2016;136(07):1031-1037

8 Giannicola G, Polimanti D, Bullitta G, Sacchetti FM, Cinotti G. Critical time period for recovery of functional range of motion after surgical treatment of complex elbow instability: prospective study on 76 patients. Injury 2014;45(03):540-545 